\title{
Editorial: Advances in Mechanical Metamaterials
}

\author{
Federico Bosia ${ }^{1}$, Anastasiia O. Krushynska ${ }^{2 *}$, Marco Miniaci ${ }^{3 *}$, Bruno Morvan ${ }^{4}$ and \\ Nicola M. Pugno ${ }^{2,5,6}$
}

${ }^{1}$ Department of Physics and Nanostructured Surfaces and Interfaces Centre, University of Turin, Turin, Italy, ${ }^{2}$ Laboratory of Bio-Inspired \& Graphene Nanomechanics, Department of Civil, Environmental and Mechanical Engineering, University of Trento, Trento, Italy, ${ }^{3}$ School of Aerospace Engineering and School of Mechanical Engineering, Georgia Institute of Technology, Atlanta, GA, United States, ${ }^{4}$ Laboratoire Ondes et Milieux Complexes, UMR CNRS 6294, University of Le Havre, Le Havre, France, ${ }^{5}$ School of Engineering \& Materials Science, Queen Mary University of London, London, United Kingdom, ${ }^{6}$ KET Labs, Edoardo Amaldi Foundation, Italian Space Agency, Rome, Italy

Keywords: metamaterials, phononic crystals, wave propagation, elasticity, acoustics

\section{Editorial on the Research Topic}

\section{Advances in Mechanical Metamaterials}

After their introduction in the field of electromagnetics at the beginning of the century, the principles of metamaterials have been applied for manipulation of elastic and acoustic waves (Deymier, 2013; Laude, 2015). In recent years, they have been the focus of research in an increasingly large community. Many fascinating effects such as cloaking, negative refraction, focusing, or the generation of band gaps have been replicated, with the peculiarity of the large range of size and frequency scales at which these can occur (Hussein et al., 2014), leading to applications in fields as diverse as ultrasonics for nondestructive testing (e.g., Miniaci et al., 2017), noise abatement (e.g., Krushynska et al., 2017), and seismic protection (e.g., Miniaci et al., 2016). In turn, this has involved the research efforts of wide and heterogeneous communities of civil engineers, physicists, mathematicians, geologists. One of the main goals of the field is presently to bring together these different research areas and to enhance cross-fertilization of ideas and approaches. This was also the aim of a recently organized workshop "Advances of Mechanical Metamaterials" held on 10-11 October, 2016, in Trento, Italy (http://www.ing.unitn.it/ pugno/news/AMM16.pdf).

This Research Topic was therefore organized to document 5 out of 24 contributions to this workshop, and more in general to provide a platform for researchers working in the field of mechanical metamaterials to disseminate their ideas on the design and characterization of new configurations, to highlight novel dynamic phenomena and explore additional promising applications, but also to stimulate the interest of other readers of the journal, with the aim of finding new potential fields of application. This is reflected in the variety of the considered structures, including meta-foundations, subwavelength rod-like resonators, tensegrity prisms, polymeric waveguides and lattices containing tilted resonators, each with different applications in mind. The contributions also span from theoretical studies to applied research.

Tallarico et al. present a study of waves in a structured geometrically chiral solid characterized by a doubly periodic high-contrast lattice containing tilted resonators. By means of Bloch-Floquet analysis, a Dirac-like dispersion band structure is identified and exploited to study wave-guiding and wave-defect interaction problems. The investigation is carried out via an adaptive finite element computation to model a transient propagation of a crack in the micro-structured material. The influence of a geometrically chiral multi-scale lattice on the field around the crack and on the edge waves propagating along structured interfaces is discussed. The authors propose these meta-structures as filters/polarizers of elastic waves and identify several potential applications. 
La Salandra et al. investigate the feasibility of a metamaterialbased foundation for seismic shielding exploiting fluid-filled tanks in industrial plants, proposing the use of so-called smart foundations. Static analysis for gravity loads is provided in accordance with current standards while maintaining favorable band-gap properties for seismic waves. A varying fluid level is considered, showing that the proposed design can attenuate vibrations in specific frequency ranges. Due to the significant impact on the attenuation efficiency of the shear stiffness of the foundation due to lateral concrete walls, an optimized design is proposed by replacing the concrete walls by less stiff concrete columns.

Miniaci et al. address the problem of generating large lowfrequency band gaps that is present in many engineering applications, from noise mitigation to vibration control and abatement of seismic wave effects. Contrary to many designs proposed so far, involving complex architectures, multiphase materials, and multiphysics, the authors propose an easy-tomanufacture design capable of opening large, low-frequency complete Lamb band gaps by exploiting a suitable arrangement of cavities in a monolithic material. The performance of the designed structure is evaluated by numerical simulations and confirmed by experimental measurements, and the design is shown to be promising for applications in which large band gaps at low frequencies are required.

Colombi et al. show how local resonance and hybridization play a key role in influencing the dispersion properties of a meta-surface made of a cluster of subwavelength resonators. The effects of rod height and cluster periodicity are investigated, showing that the former is directly related to the band-gap frequency, while the latter is related to the appearance of dynamic anisotropy. Playing with these two parameters, a gallery of meta-surfaces capable of controlling the propagation of both flexural waves in plates and surface Rayleigh waves for halfspaces is provided. Scalability with respect to the frequency and wavelength of the governing physical laws allows the application of these concepts in diverse fields and over a wide range of length scales.

\section{REFERENCES}

Deymier, P. A. (ed.). (2013). Acoustic Metamaterials and Phononic Crystals, Springer Series in Solid-State Sciences. Berlin; Heidelberg: Springer.

Hussein, M. I., Leamy, M. J., and Ruzzene, M. (2014). Dynamics of phononic materials and structures: historical origins, recent progress, and future outlook. Appl. Mech. Rev. 66:040802. doi: 10.1115/1.4026911

Krushynska, A. O., Bosia, F., Miniaci, M., and Pugno, N. M. (2017). Spider web-structured labyrinthine acoustic metamaterials for low-frequency sound control. New J. Phys. 19:105001. doi: 10.1088/1367-2630/aa83f3

Laude, V. (2015). Phononic Crystals: Artificial Crystals for Sonic, Acoustic, and Elastic Waves. Berlin; Boston, MA: Walter de Gruyter GmbH.

Miniaci, M., Gliozzi, A. S., Morvan, B., Krushynska, A., Bosia, F., Scalerandi, M., et al. (2017). Proof of concept for an ultrasensitive technique to detect and localize sources of elastic nonlinearity using phononic crystals. Phys. Rev. Lett. 118:214301. doi: 10.1103/PhysRevLett.118.214301
Modano et al. describe numerical and analytical approaches to search for freestanding configurations of class $\theta=1$ tensegrity prisms, by parameterizing the geometry of such systems in terms of two aspect angles, discussing their static and kinematic indeterminacy, and how they can be effectively stabilized through the application of pre-tensioning forces to the string members. The presence of a high number of infinitesimal mechanisms from the freestanding configurations suggests that the examined systems can be usefully employed as novel units of mechanical metamaterials that, due to their geometrically nonlinear response, may support solitary wave dynamics.

With these diverse papers, the Research Topic provides an overview of some of the most promising advances in current mechanical metamaterials research and is aimed at stimulating cross-fertilization between researchers in the field and the readers of the journal, further contributing to the emergence of this rapidly growing discipline.

\section{AUTHOR CONTRIBUTIONS}

All authors listed have made a substantial, direct and intellectual contribution to the work, and approved it for publication.

\section{ACKNOWLEDGMENTS}

We would like to thank all the authors, reviewers, and the participants of the 1st international workshop Advances of Mechanical Metamaterials held on 10-11 October, 2016, in Trento, Italy, who have supported this Research Topic. NMP is supported by the European Commission under the Graphene Flagship Core2 No. 785219 (WP14 Composites) and FET Proactive Neurofibres grant No. 732344 as well as by the Italian Ministry of Education, University and Research (MIUR) under the Departments of Excellence grant L.232/2016. FB is supported by FET Proactive Neurofibres grant No. 732344 and and by project Metapp (CSTO160004) co-funded by Fondazione San Paolo.

Miniaci, M., Krushynska, A., Bosia, F., and Pugno. N. (2016). Large scale mechanical metamaterials as seismic shields. New J. Phys. 18:083041. doi: 10.1088/1367-2630/18/8/083041

Conflict of Interest Statement: The authors declare that the research was conducted in the absence of any commercial or financial relationships that could be construed as a potential conflict of interest.

Copyright (c) 2018 Bosia, Krushynska, Miniaci, Morvan and Pugno. This is an open-access article distributed under the terms of the Creative Commons Attribution License (CC BY). The use, distribution or reproduction in other forums is permitted, provided the original author(s) and the copyright owner(s) are credited and that the original publication in this journal is cited, in accordance with accepted academic practice. No use, distribution or reproduction is permitted which does not comply with these terms. 